\title{
LINEARLY LOCALLY CONNECTED SETS AND QUASICONFORMAL MAPPINGS
}

\author{
M. F. WALKER
}

\section{Introduction}

The purpose of this paper is to study the linear local connectivity of sets in Euclidean $n$-space in relation to their boundaries, and to prove that linear local connectivity is a quasiconformal invariant which characterizes quasiconformal mappings.

The notion of linearly locally connected sets arose in the work of F. W. Gehring and J. Väisälä [7] in their investigation of quasiconformal mappings in Euclidean three-space, and appeared, under the name of strongly locally connected sets, in a paper of Gehring [3] as a means of proving that a quasiconformal mapping of a Jordan domain $D$ in three-space onto the unit ball can be extended to a quasiconformal mapping of the whole space if and only if the exterior of $D$ is quasiconformally equivalent to the unit ball. Since then, the concept has been used [4], [5], [6] in studying the univalence of analytic functions and in characterizing quasidisks.

\section{Notation}

For each integer $n \geqq 1$, let $R^{n}$ denote Euclidean $n$-dimensional space, and $\bar{R}^{n}$ the one-point compactification $R^{n} \cup\{\infty\}$. Points of $R^{n}$ will be denoted by letters such as $P, Q, x, y$. The coordinates of $x$ will be $\left(x_{1}, \ldots, x_{n}\right),|P|$ denotes the norm of $P$, and $B^{n}(P, r)$ is the ball $\{x:|x-P|<r\}$ in $R^{n}$. For a set $E \subset \bar{R}^{n}, \bar{E}, C(E)$, $\partial E$ will denote the closure, complement and boundary of $E$. If $P \in R^{n}$ and $E, F$ are sets in $\bar{R}^{n}$, then $d(P, E)$ and $d(E, F)$ denote the Euclidean distances from $E$ to $P$ and $F$ respectively. The Euclidean diameter of a bounded set $E$ is denoted by $\operatorname{diam}(E)$.

doi:10.5186/aasfm.1986.1118 


\section{Definition and examples}

3.1. Definition. A set $E \subset \bar{R}^{n}$ is c-locally connected, where $1 \leqq c<\infty$, if, for each $P \in R^{n}$ and each $r>0$, each pair of points in $E \cap \bar{B}^{n}(P, r)$ is joined by a continuum in $E \cap \bar{B}^{n}(P, c r)$, and each pair of points in $E \backslash B^{n}(P, r)$ is joined by a continuum in $E \backslash B^{n}(P, r / c)$.

$E$ is linearly locally connected if there is a number $c, 1 \leqq c<\infty$ for which $E$ is $c$-locally connected.

Quasidisks, uniform domains, the complement in $\bar{R}^{n}(n \geqq 3)$ of the quasiconformal image of a ball are all linearly locally connected sets.

It is easy to check that any linearly locally connected set is connected, locally connected at each point of $\bar{R}^{n}$ and free of cut-points. If $E$ is $c$-locally connected and $c_{1}>c, E$ is $c_{1}$-locally connected.

\section{Boundary and closure relations}

4.1. Proposition. Let $E$ be a set in $\bar{R}^{n}$. Then

(a) if $\partial E$ is c-locally connected, so is $\bar{E}$,

(b) if $E$ is c-locally connected, so is $\bar{E}$.

Proof. (a) Let $P \neq \infty$ and $r>0$ be given, and $x, y \in \bar{E} \cap \bar{B}^{n}(P, r)$. If $\bar{B}^{n}(P, r) \subset \bar{E}$, then the segment $[x, y]$ joins $x, y$ in $\bar{E} \cap \bar{B}^{n}(P, c r)$. If not, then $\partial E \cap \bar{B}^{n}(P, r)$ is non-empty, so there are (possibly trivial) segments in $\bar{E} \cap \bar{B}^{n}(P, r)$ joining $x, y$ to points $\bar{x}, \bar{y} \in \partial E \cap \bar{B}^{n}(P, r)$. The $c$-local connectivity of $\partial E$ yields a continuum $\gamma$ in $\partial E \cap \bar{B}^{n}(P, c r)$ joining $\bar{x}, \bar{y}$, so $[x, \bar{x}] \cup \gamma \cup[\bar{y}, y]$ is a continuum joining $x, y$ in $\bar{E} \cap \bar{B}^{n}(P, c r)$.

A similar argument applies to $x, y \in \bar{E} \backslash B^{n}(P, r)$.

(b) If $x, y \in \bar{E} \cap \bar{B}^{n}(P, r)$, there are sequences $\left(x_{m}\right),\left(y_{m}\right)$ in $E$ with $\lim _{m \rightarrow \infty} x_{m}=x$, $\lim _{m \rightarrow \infty} y_{m}=y$, and we may assume $x_{m}, y_{m} \in E \cap \bar{B}^{n}(P, r+1 / m)$. By the $c$-local connectivity of $E, x_{m}$ and $y_{m}$ can be joined by a continuum $\gamma_{m}$ in $E \cap \bar{B}^{n}(P, c(r+1 / m))$. Let

$$
F_{k}=\overline{\bigcup_{m=k}^{\infty} \gamma_{m}} \text { and } F=\bigcap_{k=1}^{\infty} F_{k} .
$$

Then $F_{k} \subset \bar{E} \cap \bar{B}^{n}(P, c(r+1 / k))$, and $\left(F_{k}\right)$ is a decreasing sequence of compact sets containing $x$ and $y$. Thus $F$ is a compact set in $\bar{E} \cap \bar{B}^{n}(P, c r)$ containing $x, y$.

To show $F$ is connected, we first show that, for each $\varepsilon>0, F_{k}$ is $\varepsilon$-connected for each $k \geqq k_{0}$, where $k_{0}$ is such that $\left|x-x_{m}\right|<\varepsilon$ whenever $m \geqq k_{0}$. For, if $Q_{1}, Q_{2} \in F_{k}$ and $k \geqq k_{0}$, then, for $i=1,2$ we have integers $k_{i} \geqq k$ and $Q_{i}^{\prime} \in \gamma_{k_{i}}$ with $\left|Q_{i}-Q_{i}^{\prime}\right|<\varepsilon$. As $\gamma_{k_{1}}, \gamma_{k_{2}}$ are continua, they are $\varepsilon$-connected, so there is an $\varepsilon$-chain from $Q_{1}$ to $Q_{2}$ via $Q_{1}^{\prime}, x, Q_{2}^{\prime}$ in $F_{k}$. $F_{k}$ is thus $\varepsilon$-connected.

It now follows that $F$ is $2 \varepsilon$-connected for each $\varepsilon>0$, and being compact, is a continuum joining $x, y$ in $\bar{E} \cap \bar{B}^{n}(P, c r)$. 
A similar argument applies to $x, y \in \bar{E} \backslash B^{n}(P, r)$.

Simple examples show that neither converse in Proposition 4.1 is true.

4.2. Proposition. If $E$ is a uniformly locally connected set, and $\partial E$ is c-locally connected, then $E$ is $c_{1}$-locally connected for any $c_{1}=c$.

Proof. Let $P \in R^{n}, r>0$ and $x, y \in E \cap \bar{B}^{n}(P, r)$. If the segment $[x, y]$ is in $E \cap \bar{B}^{n}(P, r)$, this is a continuum of the required type.

If not, let $x_{0}, y_{0}$ be the first and last points of $[x, y] \cap \partial E$ in passing from $x$ to $y$. As $\partial E$ is $c$-locally connected, there is a continuum $\gamma$ joining $x_{0}$ to $y_{0}$ in $\partial E \cap \bar{B}^{n}(P, c r)$

As $E$ is uniformly locally connected, for any fixed $c_{1}>c$, there is a $\delta>0$ such that, for any point $Q$ of $\gamma$, any two points of $E \cap B^{n}(Q, \delta)$ can be joined by a continuum in $E \cap B^{n}\left(Q,\left(c_{1}-c\right) r\right)$. Since $\gamma$ is a continuum, there is a $\delta / 2$-chain $x_{0}=Q_{0}, Q_{1}, \ldots, Q_{m}=y_{0}$ joining $x_{0}$ to $y_{0}$ in $\gamma$.

We use this chain to construct a continuum joining $x, y$ in $E \cap B^{n}\left(P, c_{1} r\right)$. For $i=0,1, \ldots, m$, let $\Delta_{i}=B^{n}\left(Q_{i}, \delta\right)$. Choose $P_{0} \in\left[x, x_{0}\right] \cap \Delta_{0}$. If $P_{k}$ has been chosen, let $P_{k+1} \in \Delta_{k} \cap \Delta_{k+1} \cap E$ if $k \leqq m-1$, and let $P_{m+1} \in \Delta_{m} \cap\left[y_{0}, y\right]$. By the local connectivity of $E$, there is a continuum $\gamma_{k} \subset B\left(Q_{k},\left(c_{1}-c\right) r\right) \cap E$ joining $P_{k}$ to $P_{k+1}$. Hence $\gamma_{0} \cup \ldots \cup \gamma_{m}$ is a continuum joining $P_{0}$ to $P_{m}$ in $E$, with each point less than $\left(c_{1}-c\right) r$ from $\partial E$ and so $\left[x, x_{0}\right] \cup \gamma_{0} \cup \ldots \cup \gamma_{m} \cup\left[y_{0}, y\right]$ is a continuum joining $x, y$ in $E \cap B^{n}\left(P, c_{1} r\right)$.

A similar argument applies to $x, y \in E \backslash B^{n}(P, r)$.

Remarks. 1. If, in Proposition 4.2, $E$ is a domain, the conclusion holds with $c_{1}=c$.

2. For $n \geqq 3$, there are linearly locally connected domains whose boundaries fail to be linearly locally connected.

4.3 Proposition. If $D$ is a plane domain and $\partial D$ is c-locally connected, and consists of more than one point, then $D$ is a c-locally connected Jordan domain.

Proof $^{*}$. Since $\partial D$ is connected, locally connected and free of cut-points, Theorem 9.9 of $[8$, p. 281] implies that $D$ is a Jordan domain. Thus $D$ is locally connected at each boundary point, indeed uniformly locally connected, so from Proposition 4.2 and the subsequent Remark we see that $D$ is $c$-locally connected.

4.4. Corollary. $A$ domain $D \subset \bar{R}^{2}$ is a quasidisk if and only if $\partial D$ is linearly locally connected and consists of more than one point.

Proof. This follows from a theorem of Gehring [6, p. 31] if we merely apply Proposition 4.3 when $\partial D$ is linearly locally connected.

Remark. In fact [F. W. Gehring], $D \subset \bar{R}^{2}$ is a $K$-quasicircle domain if and only if $D$ is $c$-locally connected, where $c$ depends only on $K$.

*) Dr. Kari Hag has kindly pointed out this method of proof. 


\section{Invariance under quasiconformal mappings}

We begin with [3].

5.1. Theorem. If $E$ is a c-locally connected set in $\bar{R}^{n}$ and $E^{\prime}$ is the image of $E$ under a Möbius transformation, then $E^{\prime}$ is $g(c)$-locally connected, where $g$ is the inverse of the increasing function $h$ given by

$$
h(t)=t^{1 / 2}+t^{-1 / 2}-1, \quad 1 \leqq t<\infty .
$$

For a detailed proof, see [12].

Thus linearly local connectivity is invariant under Möbius transformations.

In dealing with the effect of quasiconformal mappings, other characterizations of linear local connectivity are sometimes more convenient.

If $\Gamma$ denotes a family of locally $\sigma$-rectifiable arcs in $\bar{R}^{n}$, and $F(\Gamma)$ the family of non-negative Borel-measurable functions $\varrho$ for which $\int_{\gamma} \varrho d s \geqq 1$ for each $\gamma \in \Gamma$, we define the modulus of $\Gamma, M(\Gamma)$ by

$$
M(\Gamma)=\inf _{\gamma \in \Gamma} \int_{R^{n}} \varrho^{n} d m
$$

where $m$ denotes $n$-dimensional Lebesgue measure.

5.2. Definition. Given two sets $F_{0}, F$, in $\bar{R}^{n}$, the extremal distance, $\lambda\left(F_{0}, F_{1}\right)$, between $F_{0}, F_{1}$ is defined by

$$
\lambda\left(F_{0}, F_{1}\right)=\left(\frac{\sigma_{n-1}}{M(\Gamma)}\right)^{1 /(n-1)}
$$

where $\Gamma$ is the family of arcs joining $F_{0}, F_{1}$ in $\bar{R}^{n}$ and $\sigma_{n-1}$ is the (n-1)-dimensional measure of the unit $(n-1)$-sphere $\partial B^{n}(0,1)$.

We note that $\lambda\left(F_{0}, F_{1}\right)$ is invariant under Möbius transformations.

5.3. Definition. A set $E \subset \bar{R}^{n}$ is linearly locally connected (in the extremal distance sense) if there is a number $s>0$ such that, if $F_{0}, F_{1}$ are continua in $\bar{R}^{n}$ with

$$
\lambda\left(F_{0}, F_{1}\right) \geqq s,
$$

then $E \cap F_{i}$ can be joined by a continuum in $E \backslash F_{1-i}$, for $i=0,1$.

We relate this definition to the original (Euclidean) definition of linear local connectivity by means of rings.

An open connected set $R \subset \bar{R}^{n}$ is a ring if $C(R)$ consists of two components, which we usually denote by $C_{0}, C_{1}$. We define the modulus of a ring $R$ as

$$
\bmod R=\lambda\left(C_{0}, C_{1}\right) .
$$

If $R=\{x: a<|x-P|<b\}$ where $0<a<b<\infty$, then

$$
\bmod R=\log \frac{b}{a} .
$$


The $n$-dimensional Teichmüller ring $R_{T}(u)$ is the ring with complementary components the segment

and the ray

$$
\left\{x:-1 \leqq x_{1} \leqq 0, x_{2}=\ldots=x_{n}=0\right\}
$$

$$
\left\{x: u \leqq x_{1} \leqq \infty, x_{2}=\ldots=x_{n}=0\right\},
$$

where $u>0$. For such $u$, we define the function $\Psi_{n}$ by

$$
\log \Psi_{n}(u)=\bmod R_{T}(u) \text {. }
$$

Note that $\Psi_{n}$ is increasing and $\Psi_{n}(1)>1$. See [1] and references therein.

The link between the two definitions of linear local connectivity is provided by

5.4. Lemma. Let $F_{0}, F_{1}$ be continua in $\bar{R}^{n}$ with $\infty \in F_{1}$ and

$$
\lambda\left(F_{0}, F_{1}\right) \geqq \log \Psi_{n}(c),
$$

where $c>1$. Then for any $P \in F_{0}$, there is a number $r>0$ such that the ring $\{x: r<|x-P|<r c\}$ separates $F_{0}, F_{1}$ in $C\left(F_{0} \cup F_{1}\right)$.

Proof. Since $\lambda\left(F_{0}, F_{1}\right)>0, F_{0}, F_{1}$ are disjoint, so Lemma 3.5 of [7] implies there is a ring $R$ with complementary components $C_{0}, C_{1}$ such that, for $i=0,1$, $\partial C_{i} \subset F_{i} \subset C_{i}$. As every arc joining $F_{0}, F_{1}$ joins $C_{0}, C_{1}$ it follows easily that

$$
\lambda\left(F_{0}, F_{1}\right)=\lambda\left(C_{0}, C_{1}\right)=\bmod R .
$$

Let $r$ be the radius of the smallest ball with centre $P$ containing $C_{0}$. The extremal property of the Teichmüller ring ([10] for $R^{2}$, [2] for $R^{3}$ and [9] for $R^{n}$ ) then implies the result.

5.5. Theorem. $A$ set $E$ is linearly locally connected in the Euclidean sense if and only if $E$ is linearly locally connected in the extremal distance sense.

Proof. Suppose $E$ is linearly locally connected in the extremal distance sense, with constant $s$. Given $P \in R^{n}$ and $r>0$, let $F_{0}=\bar{B}^{n}(P, r)$ and $F_{1}=C\left(B^{i}\left(P, r e^{s}\right)\right)$. Then $\lambda\left(F_{0}, F_{1}\right)=s$, so any two points of $E \cap F_{i}$ can be joined by a continuum in $E \backslash F_{1-i}$. Therefore $E$ is $e^{s}$-locally connected.

For the converse, let $E$ be $c$-locally connected, where we may assume $c>1$, and let $F_{0}, F_{1}$ be continua in $\bar{R}^{n}$ with $\lambda\left(F_{0}, F_{1}\right) \geqq s$, where $s=\log \Psi_{n}(c)$.

Suppose first that $\infty \in F_{1}$. Choose $P \in F_{0}$ and $r$ as in Lemma 5.4, so that $\{x: r<|x-P|<r c\}$ separates $F_{0}, F_{1}$ in $C\left(F_{0} \cup F_{1}\right)$. Then $E \cap F_{0} \subset E \cap \bar{B}^{n}(P, r)$, and so is in a continuum in $E \cap \bar{B}^{n}(P, r c) \subset E \backslash F_{1}$; and $E \cap F_{1} \subset E \backslash B^{n}(P, r c)$ is in a continuum in $E \backslash B^{n}(P, r) \subset E \backslash F_{0}$.

If $\infty \notin F_{1}$, let $M$ be a Möbius transformation such that $\infty \in M\left(F_{1}\right)$. By Theorem 5.1, $M(E)$ is linearly locally connected, so the above argument applies to $M(E)$. The conclusion follows because $M^{-1}$ is a homeomorphism.

Remark. It is evident from the proofs of Lemma 5.4 and Theorem 5.5 that the definition of linear local connectivity in terms of extremal distance is equivalent 
to the analogous definition in terms of rings: a set $E \subset \bar{R}^{n}$ is linearly locally connected if and only if there is a number $s>0$ such that, for each ring $R$ with complementary components $C_{0}, C_{1}$, and $\bmod R \geqq s, E \cap C_{i}$ is in a component of $E \backslash C_{1-i}$ for $i=0,1$.

If $D, D^{\prime}$ are domains in $\bar{R}^{n}$, we say the homeomorphism $f: D \rightarrow D^{\prime}$ is a $K$-quasiconformal mapping of $D$ onto $D^{\prime}$, where $K \geqq 1$, if, for each disjoint pair of continua $F_{0}, F_{1}$ with the ring $R$ of the proof for Lemma 5.4 satisfying $\bar{R} \subset D$,

$$
\frac{1}{K} \lambda\left(F_{0}, F_{1}\right) \leqq \lambda\left(f\left(F_{0} \cap D\right), f\left(F_{1} \cap D\right)\right) \leqq K \lambda\left(F_{0}, F_{1}\right) .
$$

The mapping $f$ is quasiconformal if $f$ is $K$-quasiconformal for some $K \geqq 1$.

For $\bar{B}^{n}(P, r) \subset D$, let

$$
\begin{aligned}
L(P, r) & =\max \{|f(x)-f(P)|:|x-P|=r\}, \\
l(P, r) & =\min \{|f(x)-f(P)|:|x-P|=r\}
\end{aligned}
$$

and

$$
H(P)=\limsup _{r \rightarrow 0} \frac{L(P, r)}{l(P, r)} .
$$

Then it is known [11] that $f$ is $K$-quasiconformal if

and

$$
\sup _{P \in D} H(P)<\infty
$$

almost everywhere.

$$
H(P) \leqq K
$$

5.6. Theorem. Let $D$ be $R^{n}$ or $\bar{R}^{n}$ and $f: D \rightarrow D K$-quasiconformal mapping. If $E \subset D$ is c-locally connected, then $E^{\prime}=f(E)$ is $c^{\prime}$-locally connected, where $c^{\prime}$ depends only on $c, K$ and $n$.

Proof. If $D=R^{n}$, then we can extend $f$ to a $K$-quasiconformal mapping of $\bar{R}^{n}$ by removing the singularity at $\infty$. So we assume $D=\bar{R}^{n}$.

Let $F_{0}^{\prime}, F_{1}^{\prime}$ be continua with

$$
\lambda\left(F_{0}^{\prime}, F_{1}^{\prime}\right) \geqq K s
$$

where $s=\log \Psi_{n}(c)$. If $f^{-1}\left(F_{i}^{\prime}\right)=F_{i}, i=0,1$, then

$$
\lambda\left(F_{0}, F_{1}\right) \geqq s
$$

and so $E \cap F_{i}$ lies in a continuum in $E \backslash F_{1-i}, i=0,1$. Since $f$ is a homeomorphism, $E^{\prime} \cap F_{i}^{\prime}$ lies in a continuum in $E^{\prime} \backslash F_{1-i}^{\prime}$, for $i=0,1$. It follows that $E$ is $c^{\prime}$-locally connected, where

$$
c^{\prime}=e^{s K}=\left(\Psi_{n}(c)\right)^{K} .
$$

If the domain $D$ is a proper subset of $R^{n}$, the distance to the boundary plays a role. We first prove 
5.7. Lemma. Let $E$ be a bounded connected set in $R^{n}$ which satisfies the definition of c-local connectivity for all $P \in R^{n}$ and all $r, 0<r \leqq \delta$ where $\delta>0$ is fixed. Then $E$ is $2 c(1+d / \delta)$-locally connected, where $d=\operatorname{diam}(E)$.

Proof. Suppose $P \in R^{n}, r>\delta$ are given.

(i) If $E \cap \bar{B}^{n}(P, r) \neq \emptyset$, then the connected set

$$
E \subseteq E \cap \bar{B}^{n}(P, r+d) \subseteq E \cap \bar{B}^{n}(P,(1+d / \delta) r),
$$

so $E \cap \bar{B}^{n}(P, r)$ is in the connected set $E \cap \bar{B}^{n}(P,(1+d / \delta) r)$.

(ii) If $E \backslash B^{n}(P, r) \neq \emptyset$, then either $r \geqq 2 d$, in which case $E \subset C\left(B^{n}(P, r-d)\right) \subset$ $C\left(B^{n}(P, r / 2)\right)$, so that $E \backslash B^{n}(P, r)$ lies in the connected set $E \backslash B^{n}(P, r / 2)$; or else $\delta<r<2 d$, when $E \backslash B^{n}(P, r) \subset E \backslash B^{n}(P, \delta)$, and by hypothesis any two points of this set are joined by a continuum in $E \backslash B^{n}(P, \delta / c) \subset E \backslash B^{n}(P, r \delta /(2 c d))$.

The result now follows readily.

5.8. Theorem. Let $D, D^{\prime}$ be proper subdomains of $R^{n}$, and $f: D \rightarrow D^{\prime}$ a $K$-quasiconformal mapping. If $E$ is a c-locally connected set with $\bar{E} \subset D$, then $E^{\prime}=f(E)$ is $c^{\prime}$-locally connected, where $c^{\prime}=c^{\prime}(c, K$, diam $(E) / d(E, \partial D), n)$.

Proof. We first exhibit numbers $c^{\prime \prime}>1$ and $\delta^{\prime}>0$ for which Lemma 5.7 applies to $E^{\prime}$.

Since $E^{\prime} \subset D^{\prime}, E^{\prime}$ is bounded and $0<d\left(E^{\prime}, \partial D^{\prime}\right)<\infty$. Let $c^{\prime \prime}=\left(\Psi_{n}(c)\right)^{K}$, and choose $\delta^{\prime}<d\left(E^{\prime}, \partial D^{\prime}\right) /\left(c^{\prime \prime}+1\right)$.

Let $P^{\prime} \in R^{n}$, and $0<r^{\prime} \leqq \delta^{\prime}$. If $E^{\prime} \cap \bar{B}^{n}\left(P^{\prime}, r^{\prime}\right) \neq \emptyset$ then $d\left(P^{\prime}, E^{\prime}\right) \leqq r^{\prime}$, and so

$$
d\left(\partial D^{\prime}, \bar{B}^{n}\left(P^{\prime}, r^{\prime} c^{\prime \prime}\right)\right) \geqq d\left(\partial D^{\prime}, E^{\prime}\right)-\left(c^{\prime \prime}+1\right) r^{\prime}>0 .
$$

Hence $\bar{B}^{n}\left(P^{\prime}, r^{\prime} c^{\prime \prime}\right) \subset D^{\prime}$. Denoting by $R^{\prime}$ the ring $\left\{x: r^{\prime}<\left|x-P^{\prime}\right|<c^{\prime \prime} r^{\prime}\right\}$, we see that $R=f^{-1}\left(R^{\prime}\right)$ has modulus at least

$$
\frac{1}{K} \bmod R^{\prime}=\frac{1}{K} \log c^{\prime \prime}=\log \Psi_{n}(c) .
$$

But if $R$ has complementary components $C_{0}, C_{1}$, where $C_{0}=f^{-1}\left(\bar{B}^{n}\left(P^{\prime}, r^{\prime}\right)\right)$, we see $E \cap C_{i}$ is in a continuum in $E \backslash C_{1-i}$ for $i=0,1$. Since $f$ is a homeomorphism, $E^{\prime} \cap \bar{B}^{n}\left(P^{\prime}, r^{\prime}\right)$ is in a component of $E^{\prime} \cap \bar{B}^{n}\left(P^{\prime}, r^{\prime} c^{\prime \prime}\right)$, and $E^{\prime} \backslash B^{n}\left(P^{\prime}, r^{\prime} c^{\prime \prime}\right)$ is in a component of $E^{\prime} \backslash B^{n}\left(P^{\prime}, r^{\prime}\right)$.

Lemma 5.7 now shows $E$ is $c^{\prime}$-locally connected, where $c^{\prime}$ depends on $c, K$ and $\operatorname{diam} E^{\prime} / d\left(E^{\prime}, \partial D^{\prime}\right)$.

It now suffices to show that diam $E^{\prime} / d\left(E^{\prime}, \partial D^{\prime}\right)$ depends only on $K$, $\operatorname{diam} E / d(E, \partial D)$ and $n$. For this, let $\alpha$ be the number such that

$$
\Theta_{K}^{n}(\alpha)=\frac{1}{3}
$$


where $\Theta_{K}^{n}$ is the distortion function of [11, p. 63], and let $\delta=d(E, \partial D)$. Consider a grid of closed $n$-cubes $Q$ meeting $E$, with disjoint interiors, of side-length $\alpha \delta /((\alpha+1) \sqrt{n})$. The number of such cubes clearly cannot exceed a bound depending only on $n, K$ and $\operatorname{diam}(E) / d(E, \partial D)$. If $x, y \in Q$, then

so that

$$
d(Q, \partial D) \geqq d(x, \partial D)-\operatorname{diam} Q \geqq \delta-\frac{\alpha \delta}{\alpha+1}=\frac{\delta}{\alpha+1}
$$

$$
\frac{|x-y|}{d(x, \partial D)} \leqq \frac{\operatorname{diam} Q}{d(Q, \partial D)} \leqq \alpha .
$$

Denoting $f(x), f(y), f(Q)$ by $x^{\prime}, y^{\prime}, Q^{\prime}$, we have

$$
\frac{\left|x^{\prime}-y^{\prime}\right|}{d\left(x^{\prime}, \partial D^{\prime}\right)}, \frac{\left|x^{\prime}-y^{\prime}\right|}{d\left(y^{\prime}, \partial D^{\prime}\right)} \leqq \Theta_{K}^{n}\left(\frac{|x-y|}{d(x, \partial D)}\right) \leqq \Theta_{K}^{n}(\alpha)=\frac{1}{3},
$$

by Theorem 18.1 of [11]. This implies

$$
\frac{\operatorname{diam} Q^{\prime}}{d\left(Q^{\prime}, \partial D^{\prime}\right)} \leqq \frac{1}{2} \text {. }
$$

Since $E$ is connected, there is a simple arc $\gamma$ joining points $x, y$ in $E$ with $|f(x)-f(y)|=\operatorname{diam}\left(E^{\prime}\right)$. Clearly, $\gamma$ can be covered by a sequence $Q_{1}, \ldots, Q_{N}$ of cubes of the grid such that $x \in Q_{1}, y \in Q_{N}$ and $F_{k} \cap Q_{k+1} \neq \emptyset$ where $F_{k}=\bigcup_{j=1}^{k} Q_{j}$. Let $F_{k}^{\prime}=f\left(F_{k}\right)$. Simple geometric considerations show that

$$
\frac{\operatorname{diam}\left(F_{k}^{\prime}\right)}{d\left(F_{k}^{\prime}, \partial D^{\prime}\right)} \leqq m_{k}, \quad k=1, \ldots, N,
$$

where $m_{1}=1 / 2$ and $m_{k+1}=3 m_{k} / 2+1 / 2, k=1, \ldots, N-1$. But then

$$
\frac{\operatorname{diam}\left(E^{\prime}\right)}{d\left(E^{\prime}, \partial D^{\prime}\right)} \leqq \frac{\operatorname{diam}\left(F_{N}^{\prime}\right)}{d\left(F_{N}^{\prime}, \partial D^{\prime}\right)} \leqq m_{N},
$$

a number with an upper bound depending only on $n, K$ and $\operatorname{diam}(E) / d(E, \partial D)$. The proof of Theorem 5.8 is complete.

5.10. Corollary. Let $f$ be a quasiconformal mapping of the domain $D$ onto the domain $D^{\prime}$ in $\bar{R}^{n}$. If $E$ is a linearly locally connected set with $\bar{E} \subset D$, then $f(E)$ is linearly locally connected.

Proof. This follows from Theorem 5.6, Theorem 5.8 and the invariance of linear local connectivity under Möbius transformations.

We have the following characterization of quasiconformal mappings:

5.11. Theorem. Let $f$ be a homeomorphism of a domain $D$ onto a domain $D^{\prime}$ in $\bar{R}^{n}$. If $f$ maps each c-locally connected set $E$ with $\bar{E} \subset D \backslash\{\infty\}$ onto a $c^{\prime}$-locally connected set where $c^{\prime}$ depends only on $c, f$ and $\operatorname{diam}(E) / d(E, \partial D)$ (in case $R^{n} \subset D$, we take the last to be 0$)$, then $f$ is a quasiconformal mapping of $D$ onto $D^{\prime}$. 
Proof. Write $D_{1}=D \backslash\left\{\infty, f^{-1}(\infty)\right\}$ and let $P \in D_{1}$. Choose $a$ so that $0<a<$ $d\left(P, \partial D_{1}\right) / 2$, where the last may be $\infty$. For $0<r<a / 2$, let $P_{1}, P_{2} \in \partial B^{n}(P, r)$ so that the acute angle between the segments $P P_{1}, P_{1} P_{2}$ is at least $\pi / 4$, and let $P_{3}$, $P_{4}$ be the points of intersection of the rays $P_{1} P, P_{1} P_{2}$ with $\partial B^{n}(P, a)$. The set $E$ consisting of the segments $P_{1} P_{3}, P_{4} P_{1}$ and the minor arc of the great circle of $\partial B^{n}(P, a)$ through $P_{3}, P_{4}$ is then $\operatorname{cosec} \pi / 8$-locally connected, and, by choice of $a$, $\operatorname{diam}(E) / d(E, \partial D)<1 / 2$. Then $f(E)$ is $c^{\prime}$-locally connected, where $c^{\prime}$ is independent of $P$ and the particular choice of $E$.

Now, if $r$ is so small that

$$
\frac{1}{2}\left(c^{\prime}+1\right) L(P, r) \leqq l(P, a),
$$

it is not difficult to show that

$$
\left|f(P)-f\left(P_{1}\right)\right| \leqq \frac{1}{2}\left(c^{\prime}+1\right)\left|f(P)-f\left(P_{2}\right)\right|
$$

from the $c^{\prime}$-local connectivity of $f(E)$. But then it follows that

so that

$$
L(P, r) \leqq \frac{1}{4}\left(c^{\prime}+1\right)^{2} l(P, r)
$$

for all points $P \in D_{1}$.

$$
H(P)=\limsup _{r \rightarrow 0} \frac{L(P, r)}{l(P, r)} \leqq \frac{1}{4}\left(c^{\prime}+1\right)^{2}
$$

It follows that $\left.f\right|_{D_{1}}$ is quasiconformal. But then $f$ is quasiconformal on $D$, since if $\infty \in D$ or $D^{\prime}, \infty, f^{-1}(\infty)$ are removable singularities.

Remark. We have only used the hypothesis of Theorem 5.11 when $c=\operatorname{cosec}(\pi / 8)$. When $n=2$, this can be sharpened to $c=1$.

I wish to thank Professor F. W. Gehring for his encouragement to write this paper.

\section{References}

[1] Alsderson, G. D.: Extremal rings in $n$-space for fixed and varying $n$. - Ann. Acad. Sci. Fenn, Ser. A I Math. 575, 1974, 1-21.

[2] Gehring, F. W.: Symmetrization of rings in space. - Trans. Amer. Math. Soc. 101, 1961, 499-519.

[3] Gehring, F. W.: Extension of quasiconformal mappings in three-space. - J. Analyse Math. $14,1965,171-182$

[4] Gehring, F. W.: Quasiconformal mappings of slit domains in three-space. - J. Math. Mech. $18,1969,689-703$.

[5] GeHring, F. W.: Univalent functions and the Schwarzian derivative. - Comment. Math. Helv. $52,1977,561-572$.

[6] Gehring, F. W.: Characteristic properties of quasidisks. - Séminaire de Mathématiques Supérieures 84. Presses de l'Université de Montréal, Montréal, Que., 1982. 
[7] GeHrING, F. W., and J. VÄIsÄLÄ: The coefficients of quasiconformality of domains in space. Acta Math. 114, 1965, 1-70.

[8] Pommerenke, C.: Univalent functions. - Vandenhoeck \& Ruprecht, Göttingen, 1975.

[9] Sarvas, J.: Symmetrization of condensers in $n$-space. - Ann. Acad. Sci. Fenn. Ser. A I Math. $522,1972,1-44$.

[10] TeIChMÜLLER, O.: Untersuchungen über konforme und quasikonforme Abbildung. - Deutsche Math. 3, 1938, 621-678.

[11] VÄISÄLÄ, J.: Lectures on $n$-dimensional quasiconformal mappings. - Lecture Notes in Mathematics 229. Springer-Verlag, Berlin-Heidelberg-New York, 1971.

[12] WALKER, M. F.: Quasiconformal mappings and strong local connectivity. - Dissertation, University of Michigan, 1969.

University of Cape Town

Department of Mathematics

Rondebosch, C.P.

7700 , Republic of South Africa

Received 24 April 1984 